\title{
Therapeutic Role of MiR-140-5p for the Treatment of Non-small Cell Lung Cancer
}

\author{
VALENTINA FLAMINI, WEN G. JIANG and YUXIN CUI \\ Cardiff China Medical Research Collaborative, Cardiff University School of Medicine, Cardiff, U.K.
}

\begin{abstract}
Background/Aim: Lung cancer is the second most common cancer in both men and women, after prostate and breast cancer, respectively. There are two main types of primary lung cancer, non-small cell lung cancer (NSCLC), which accounts for approximately 85-90\% of all lung cancer cases, and small cell lung cancer (SCLC), which accounts for the other 10-15\% of lung cancers. MiRNAs are small molecules that post-transcriptionally regulate many genes and contribute to many disease aetiologies, including tumours. In lung cancer, the down-regulation of miR-140-5p leads to disease progression. Materials and Methods: In this study a miR-140-5p-only treatment and miR-140-5p combined with other chemotherapeutics have been studied in vitro. Results: When transfected into NSCLC, the overexpression of miR-140-5p reduced the migration and invasion properties of malignant cells and, also improved their adhesion onto the artificial extracellular matrix. When miRNA-140-5p replacement treatment was combined with other drugs commonly used in clinical practice, such as gefinitib, DMHI and cisplatin, it enhanced their efficacy by reducing the migration and invasion ability of cancer cells, thus suggesting that it acts synergistically with known compounds for the treatment of NSCLC. Additionally, some endothelial mesenchymal transition (EMT) markers appeared to be regulated by miR-140-5p. Conclusion: Novel direct targets of miR-140-5p have not been investigated in this study, but our results indicate the involvement of miR$140-5 p$ in lung cancer invasion. The preliminary data from this study imply that when miR-140-5p levels are restored; maybe synergistically support current therapies for NSCLC though further validation, especially in vivo is required.
\end{abstract}

Correspondence to: Dr. Yu-Xin Cui, Cardiff China Medical Research Collaborative, Henry Wellcome Building, School of Medicine, Cardiff University, Heath Park, Cardiff, CF14 4XN, U.K. Tel: +44 02920687070, e-mail: CuiY7@cardiff.ac.uk

Key Words: MiR-140-5p, miRNA replacement treatment, lung cancer cells, invasion.
Lung cancer is the most frequently diagnosed cancer worldwide, with 46,403 new cases diagnosed in the UK in 2014. The next two most common types are breast (11.9\%) and colorectal cancer $(9.7 \%)(1,2)$. Historically, lung cancers from the cells of respiratory epithelium have been broadly classified into two types based on the microscopic appearance of the tumour cells: small lung cancers (SCLC) and non-small cell lung cancers (NSCLC). SCLC comprises about $10-15 \%$ of lung cancer cases, is the most aggressive and strongly related to cigarette smoking (2). On the other hand, NSCLC is the most common type, accounting for about $85-90 \%$ of all cases, and has a high tendency to spread to the lymph nodes and distant sites.

MicroRNAs (miRNAs or miRs) are small non-coding RNAs that influence the pathogenesis of human diseases, including cancer, by binding their target messenger RNAs and thus preventing protein translation. Many microRNAs have been shown to be up- or down-regulated in many human malignancies, suggesting they have roles as either oncogenes or tumour suppressors (3). MiR-140 precursor is located on chromosome 16q22.1 and produces two different mature single-strand molecules named 140-3p and 140-5p, which have multiple targets and are down-regulated in several cancers, including $\operatorname{NSCLC}(4,5)$. It has been demonstrated that low levels of miR-140-5p promote the progression of many malignancies and this indicates its function as a likely tumour-suppressor (4, 6-9). Most miRNAs are down-regulated in NSCLC and other cancers, and therapies aimed at miRNA replacement have been successful in both in vitro and in vivo models. Nanoparticles have been used in cancer for the systemic delivery of miRNA and siRNA without toxicity in several tumour types, including lung cancer $(10,11)$. Although this methodology still represents a challenge in cancer therapy, the present results are promising. One of the most appealing properties of miRNAs as therapeutic agents is their ability to target multiple molecules. For instance miR-181 regulates T-cell receptor sensitivity targeting multiple phosphatases, an effect that was not observed by silencing a single component of the pathway by siRNA (12). This ability to target multiple 
transcripts, together with their low molecular weight, provides a new opportunity for cancer treatment compared to other methods, such as gene therapy.

In the present study we investigated the effect of miR140-5p on NSCLC behaviour and studied its ability to act synergistically with other anticancer therapies specific for the treatment of lung cancer patients with mutations in the endothelial growth factor (EGFR) and activin receptor-like kinase 2 (ALK2) receptor. The effect on cancer cell behaviour of the combined miRNA mimics treatment and commercially available NSCLC chemotherapeutic agents has been evaluated by using EGFR and ALK2 inhibitors, as the mutations in these genes are very common in both smoking and non-smoking NSCLC patients. Cisplatin has also been included because of its wide clinical application in the treatment of tumours, including lung (13).

\section{Materials and Methods}

Cell culture and tissues. A549 carcinoma and SK-MES1 squamous carcinoma NSCLC cell lines were obtained from Lonza (Gloucestershire, UK) and maintained in Dulbecco's modified Eagle's medium (DMEM, GIBCO,USA) with $10 \%$ fetal bovime serum (FBS) and antibiotics (100U/ml). The normal lung cell line BEAS-2B was maintained in Bronchial Epithelial Cell Growth Medium (BEBM, Lonza, Gloucestershire, UK) supplemented with manufacturer's recommended additives $\left(\mathrm{BEGM}^{\mathrm{TM}}\right.$ BulletKit, Lonza, Gloucestershire, UK). All tissues from NSCLC patients were collected immediately after surgery by Capital Medical University Hospital, Beijing, China and stored at $-80^{\circ} \mathrm{C}$ until use, with approval of the Health Authority local research ethics committee. The cohort included 19 paired tumour and adjacent normal lung tissues (Table I). Paired tissues refer to tissues from the same patients, in which the tumour part and the adjacent normal counterpart have been resected surgically. All the specimens used in the current study were verified by a consultant pathologist.

MiRNA treatment. Hsa-miR-140-5p and a scramble control were purchased from Sigma (MISSION ${ }^{\circledR}$ Human miRNA Mimics, Sigma-Aldrich, Dorset, UK). Mimics and control were transfected into cells at a final concentration of $20 \mu \mathrm{M}$ using $2 \mu \mathrm{l} / \mathrm{ml}$ of DermaFect 1 transfection reagents (Thermo Fisher Scientific, Waltham, MA USA) in antibiotic-free medium.

Quantitative real-time polymerase chain reaction ( $q R T-q P C R$ ). Total RNA was extracted from all the cell lines and tissues using TRI reagent (Sigma-Aldrich, Dorset, UK) according to the manufacturer's instructions. The total RNA was converted into cDNA using miRNA-specific reverse and forward primers (SigmaAldrich, Dorset, UK) (Table II) and the Reverse Transcription (cDNA Synthesis) Products (New England BioLabs ${ }^{\circledR}, \mathrm{UK}$ ). The conditions used for reverse transcription were $42^{\circ} \mathrm{C}$ for $60 \mathrm{~min}$ and $95^{\circ} \mathrm{C}$ for 5 min using Applied Biosystems 2720 Thermal Cycler (Life Technologies, Paisley, UK). Following reverse transcription, cDNA was diluted 1:24 using DEPC water. PCR amplification was performed using the SYBR Green JumpStart Taq ReadyMix (Sigma-Aldrich, Dorset, UK) and miRNA-specific reverse and forward primers (Sigma-Aldrich, Dorset, UK) in a IQ5 Bio-Rad
Table I. Non-small cell lung cancer cohort characteristics. The cohort included a total of 19 paired tissues.

\begin{tabular}{lccc}
\hline & Characteristic & Number & Percentage \\
\hline \multirow{2}{*}{ Gender } & Female & 3 & $16 \%$ \\
& Male & 11 & $57 \%$ \\
\multirow{4}{*}{ TNM } & Unknown & 5 & $27 \%$ \\
& T1N2M0 & 1 & $5 \%$ \\
& T1N2M1 & 1 & $5 \%$ \\
& T2N2M0 & 5 & $27 \%$ \\
& T2N3M0 & 1 & $5 \%$ \\
& T3N0M0 & 1 & $5 \%$ \\
& PT3N2M0 & 1 & $5 \%$ \\
Smoking history & Unknown & 9 & $48 \%$ \\
& Non-smokers & 2 & $10 \%$ \\
& Smokers & 13 & $69 \%$ \\
& Unknown & 4 & $21 \%$ \\
\hline
\end{tabular}

qPCR thermocycler. Reaction used the following parameters: predenaturation for $10 \mathrm{~min}$ at $94^{\circ} \mathrm{C}$; followed by 40 cycles of denaturation at $94^{\circ} \mathrm{C}$ for $15 \mathrm{sec}$, annealing and elongation at $58^{\circ} \mathrm{C}$ for $1 \mathrm{~min}$. Results were analysed using 2-( $\Delta \Delta \mathrm{CT})$ method of normalisation to housekeeping gene U6 in cell lines and U48 in tissues. A melting curve analysis was also included.

Proliferation assay. A549 and SK-MES- $1\left(5 \times 10^{3}\right.$ cells/well) cells were seeded onto 96-well plates in $100 \mu \mathrm{l}$ growth medium. Following 24 and $48 \mathrm{~h}$ of miRNA and/or drug treatment, the transfection medium was replaced with complete medium and the cells were fixed by using $100 \mu \mathrm{l}$ formalin for $15 \mathrm{~min}$, before being stained with $100 \mu 10.5 \%$ Crystal Violet for a further $15 \mathrm{~min}$. The dye was then washed off with water and the plates left to dry overnight. The day after, $200 \mu \mathrm{l}$ of acetic acid were added into each well and the absorbance was measured at $540 \mathrm{~nm}$ using a spectrophotometer (BIO-TEK, Elx 800, UK).

Transwell migration and invasion assays. Cells were detached using HYQTase Cell Detachment Solution (Hyclone, Logan, UT, USA) 24 $\mathrm{h}$ after being transfected and resuspended in serum-free media, at a concentration of $1 \times 10^{5}$ cells $/ \mathrm{ml}$. On the bottom of each well, $650 \mu 1$ of medium containing 10\% FCS (chemoattractant) or serum-free media (no chemoattractant) were added. Policarbonate $8 \mu \mathrm{m}$ pore sized ThinCert ${ }^{\mathrm{TM}}$ 24-well plate inserts (Grenier, Bio-One $\mathrm{GmbH}$, Austria) were placed in each well and $500 \mu$ of cell suspension added. For invasion assay chambers were coated with serum-free medium containing Matrigel (BD Biosciences, NJ USA) diluted in serum-free medium to gain a concentration of $500 \mu \mathrm{g} / \mathrm{ml}$. After 24 $\mathrm{h}$ incubation at $37^{\circ} \mathrm{C}$, the inserts were washed with PBS and then incubated for $1 \mathrm{~h}$ at $37^{\circ} \mathrm{C}$ in $350 \mu \mathrm{l}$ Cell Dissociation Solution (CDS; Sigma Aldrich, Dorset, UK)/Calcein AM (eBioscience, Hatfield, UK) at a ratio of $1.2 \mu \mathrm{l}$ Calcein AM in $1 \mathrm{ml}$ CDS. The cells suspension was then transferred into a black 96-well plate and fluorescence was measured (excitation $485 \mathrm{~nm} /$ emission $520 \mathrm{~nm}$ ) using Glomax Multi Detection System (Promega, Wisconsin, USA). To analyse the total directed cell movement, the wells containing no chemoattractant was subtracted from the test wells. 
Table II. $R T-q P C R$ primers.

\begin{tabular}{lll}
\hline Primer & & Sequence \\
\hline miR-specific RT-primer & & CAGGTCCAGTTTTTTTTTTTTTTTVN* \\
RNU6B & Forward & GTCGTGAAGCGTTCCA \\
& Reverse & CAGGTCCAGTTTTTTTTTTTTTTAAA \\
miR $140-5 \mathrm{p}$ & Forward & CAGCAGTGGTTTACCCTATG \\
& Reverse & GGTCCAGTTTTTTTTTTTTTCTAC \\
miR $140-3 \mathrm{p}$ & Forward & GTACCACAGGGTAGAACCA \\
& Reverse & GTACCACAGGGTAGAACCA \\
GAPDH & Forward & AAGGTCATCCATGACAACTT \\
& Reverse & ACTGAACCTGACCGTACAATCGCTCCACCAACTAAGAAC \\
\hline
\end{tabular}

$* \mathrm{~V}=\mathrm{A}$ or $\mathrm{G}$ or $\mathrm{C} ; \mathrm{N}=\mathrm{A}$ or $\mathrm{G}$ or $\mathrm{C}$ or $\mathrm{T}$.

Adhesion assay. A total of $1 \times 10^{4}$ transfected cells/well were added into a 96-well plate coated with $100 \mu \mathrm{g} /$ well of laminin or collagen. A549 and SK-MES1 were allowed to attach at $37^{\circ} \mathrm{C}$ for 40 and $60 \mathrm{~min}$, respectively, then washed with PBS, fixed using $200 \mu \mathrm{l}$ of $4 \%$ Formalin and incubated at room temperature for $15 \mathrm{~min}$. Crystal Violet $0.5 \%$ was then added into each well, the plate was incubated again at room temperature for $10 \mathrm{~min}$, washed twice with distilled water and finally left overnight at $37^{\circ} \mathrm{C}$ to dry. The following day the absorbance was measured using Glomax Multi Detection System (Promega, Wisconsin, USA).

Drug treatment. After $24 \mathrm{~h}$ post-transfection, cells were treated with anti-cancer therapeutics for a further $24 \mathrm{~h}$. Control cells were incubated with DMSO. The drugs used were gefinitib (Tocris, Abingdon, UK), an inhibitor of EGFR, at a concentration of $1 \mu \mathrm{M}$, DMH1 (Selleckchem, Munich, Germany), an inhibitor of ALK2, at a concentration of $0.1 \mathrm{M}$ and Cisplatin (TEVA, Castelford, UK) at a concentration of $2 \mu \mathrm{M}$.

Cell-cycle assay. Cells were washed twice with PBS and detached using HYQTase Cell Detachment Solution (Hyclone, Logan, UT, USA) 24 ho after being transfected and resuspended in serumcontaining medium. Cells were centrifuged twice at $17,000 \mathrm{~g}$ for $5 \mathrm{~min}$. The first time, cells were resuspended in $5 \mathrm{ml}$ of ice cold PBS and the second time in $1 \mathrm{ml}$ of ice cold $70 \% \mathrm{v} / \mathrm{v}$ ethanol. Next, cells were incubated on ice for 2 ho, centrifuged again at 17,000g for $5 \mathrm{~min}$ and concentrated at $1 \times 10^{6}$ cells in $100 \mu \mathrm{l}$ of PBS for each sample. Except for the non-stained controls, $150 \mu \mathrm{l}$ of $\mu \mathrm{g} / \mathrm{ml}$ propidium iodide (PI; ThermoFisher) was added into each sample and the cells were further incubated at room temperature in the dark for 15 min. Following this, samples were run on the BD FACSCanto ${ }^{\mathrm{TM}}$ II flow cytometer (BD) using the FL3 channel (575 nm).

Western Blotting. Following $48 \mathrm{~h}$ of treatment with miR-140-5p mimics, cells were lysed using RIPA buffer. The protein concentration was determined, an equal volume of $2 x$ Lamllie buffer (Sigma Aldrich, Dorset, UK) was added and the mixture was heated to $95^{\circ} \mathrm{C}$ for $5 \mathrm{~min}$ and loaded on a gel. Gel was run for $2.5 \mathrm{~h}$ at $100 \mathrm{~V}$. Gel was transferred onto PVDF membrane using wet transfer for $50 \mathrm{~min}$, at $500 \mathrm{~mA}$. The membrane was blocked in $5 \%$ milk for $1 \mathrm{~h}$, then probed overnight with the primary antibodies. All the primary antibodies used in this study were purchased from Santa Cruz Biotechnology (Santa Cruz, Texas, USA). All the antibodies were diluted 1:200, except GAPDH that was diluted 1:1000. Membranes were washed three times in TBS-Tween $(0.01 \%)$ and then probed with the appropriate secondary antibody purchased from Sigma-Aldrich (Dorset, UK) and diluted 1:1,000. Protein bands were detected using EZ-ECL (Biological Industries, Staffordshire, UK) and visualised using a G:BOX (Syngene, Cambridge, UK).

Electric Cell-Substrate Impedance Sensing (ECIS) wound-healing assay. The Electric Cell-Substrate Impedance Sensing (ECIS) system was used to monitor, in real-time, the motility of the cancer cells transfected with the miRNA mimics with and without drug treatments. Cancer cells were seeded in 96WE ECIS plates at a density of $1.5 \times 10^{4}$ cells/well and left overnight. The day after, they were transfected with miRNA mimics and in the following $24 \mathrm{~h}$ treated with the chemotherapy drugs. Following a further $24 \mathrm{~h}$, the media were replaced with serum-containing media and the assay performed. The resistance of the monolayer was monitored at a frequency of $64,000 \mathrm{~Hz}$ before and after electrical wounding.

Statistical analysis. Pearson-D'Agostino test was used to verify if the data were normally distributed. Unpaired or paired t-test was used for normal distributions. For non-normal distributions, the Mann-Whitney Rank Tests was used for the unpaired samples and Wilcoxon-Test for the paired ones. Welch's correction was applied in the unpaired $t$-test. Graphs and the statistical analysis were performed using GraphPad Prism 6.04 software (GraphPad Software, San Diego, CA, USA) or Excel 2015. Statistical significance was indicated with the following nomenclature: $* p<0.05, * * p<0.01, * * * p<0.001$.

\section{Results}

MiR-140-5p is down-regulated in NSCLC tissues and cell lines and higher levels were restored by the miRNA replacement treatment. The expression of miR-140-5p was assessed in the two NSCLC cell lines A549 and SK-MES1 and in the immortalised normal lung epithelial cell line BEAS-2B. MiR-140-5p was significantly down-regulated in the cancer cell lines compared to the normal one, with $p=0.0018$ and $p=0.0014$ in A549 and SK-MES1 respectively (Figure 1a). In paired tissues, the results observed in the cell 

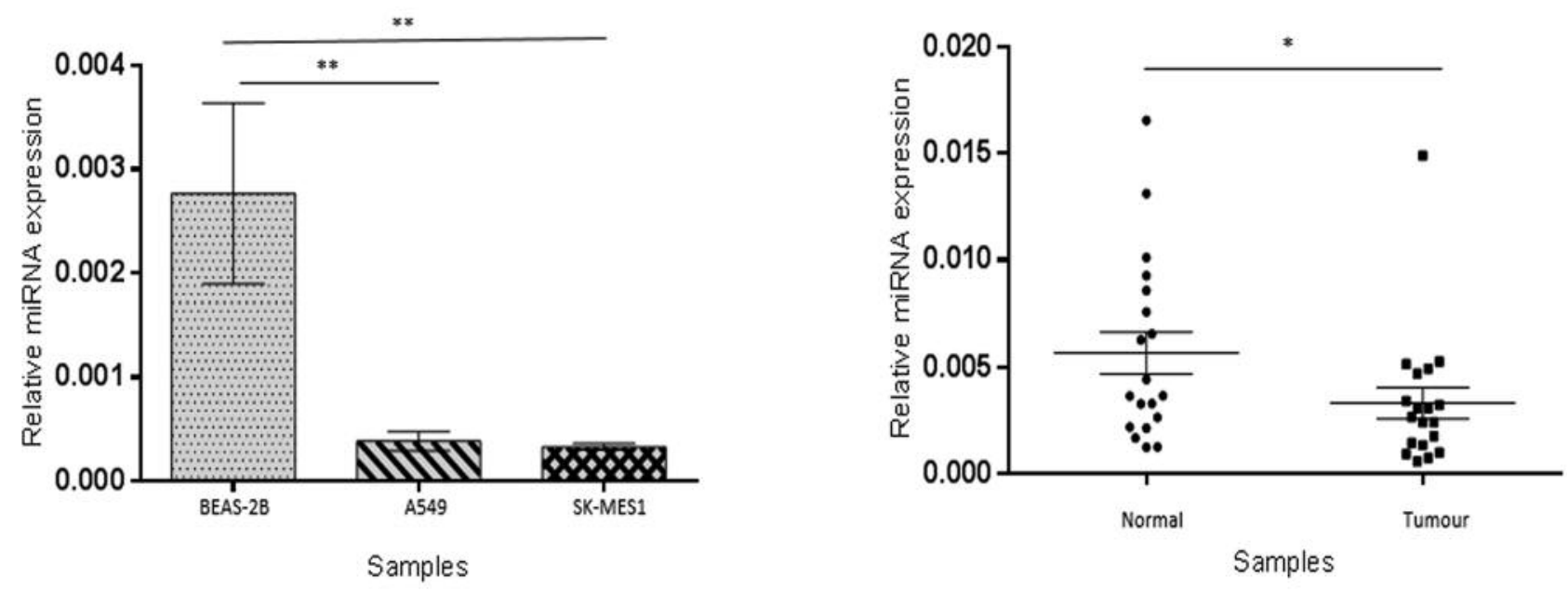

Figure 1. MiR-140-5p expression in lung cancer cell lines and tissues and miR-140-5p efficiency of transfection in cancer cell lines. a) MiR-140$5 p$ is down-regulated in A549 and SK-MES1 cancer cell lines compared to the normal epithelial cell line BEAS-2B. b) MiR-140-5p expression was lower in lung cancer tissues, when compared to their normal adjacent counterpart. MiR-140-5p expression in cancer cell lines and tissues was evaluated by a miR-specific RT-qPCR. Data are presented as mean \pm SEM of three individual experiments undertaken in triplicate. T-test for paired data with Welch's correction or Wilcoxon-Rank test have been used to assess significance for paired tissues with *p<0.05, **p<0.01.

lines was replicated with a lower expression of miR-140-5p $(p=0.0428)$ observed in cancer tissues compared to the adjacent normal counterparts (Figure 1b). MiR-140-5p levels significantly increased in A549 and SK-MES1 cells after transfection with the miR-140-5p mimics (Figure 2). The fold-change of miR-140-5p levels in A549 cells treated with the mimics were $12100(p<0.0001)$ and $943(p<0.0001)$ after 24 and $48 \mathrm{~h}$, respectively. The transfection with miR-140-5p mimics also led to a significant increase of the miRNA levels in SK-MES1 cells, with a fold change of $1,204(p<0.0001)$ and $4628(p=0.002)$ after 24 and $48 \mathrm{~h}$ respectively.

MiR-140-5p overexpression inhibited the lung cancer progression in vitro. MiR-140-5p overexpression did not have any effect on the proliferative ability and cell cycle of the two NSCLC cell lines (Figure 3a and b). However, a significant reduction of other malignant characteristics was observed (Figure $3 \mathrm{c}$ and d). Forty-eight $\mathrm{h}$ after transfection with miR-140-5p mimics, a reduction in the migratory properties of SK-MES1 cells $(p=0.025)$ was observed (Figure 3c). MiR-140-5p also significantly decreased the invasive potential of both NSCLC cells, $p=0.0036$ in A549 and $p<0.001$ in SK-MES1 (Figure 3c). To test whether miR140-5p had any effect on the cancer cell adhesion to an artificial extracellular matrix (ECM), the transfected cells were seeded on laminin or collagen, two major ECM basal lamina protein components. A549 adhesion significantly increased in transfected cells cultured onto laminin $(p=0.0137)$ and collagen $(p=0.0076)$ layers. No effect on SK-MES1 cell adhesion was observed (Figure 3d).

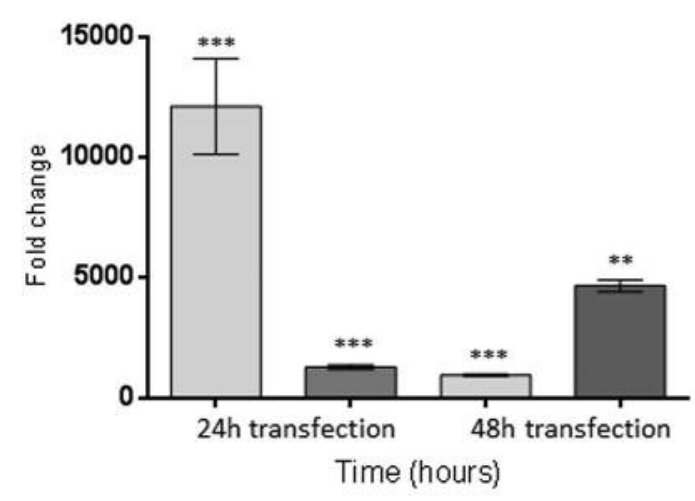

A549

SK-MES1

Figure 2. MiR-140-5p levels after transfection. MiR-140-5p mimics increased the level of the miRNA in vitro, in A549 and SK-MES1 cell lines in a time-dependent manner. Data are presented as mean \pm SEM, three individual experiments undertaken in triplicate. T-test with Welch's correction or Wilcoxon-Rank test were used to assess significance with $* * p<0.01, * * * p<0.001$.

A combined treatment with miR-140-5p mimics and chemotherapeutics affects the proliferation and migration ability of NSCLC in vitro. The effect on cancer cell behaviour under the combined treatment of miRNA mimics and some chemotherapeutic agents commercially available for the treatment of the NSCLC, has been investigated in this study. EGFR is constitutively activated 

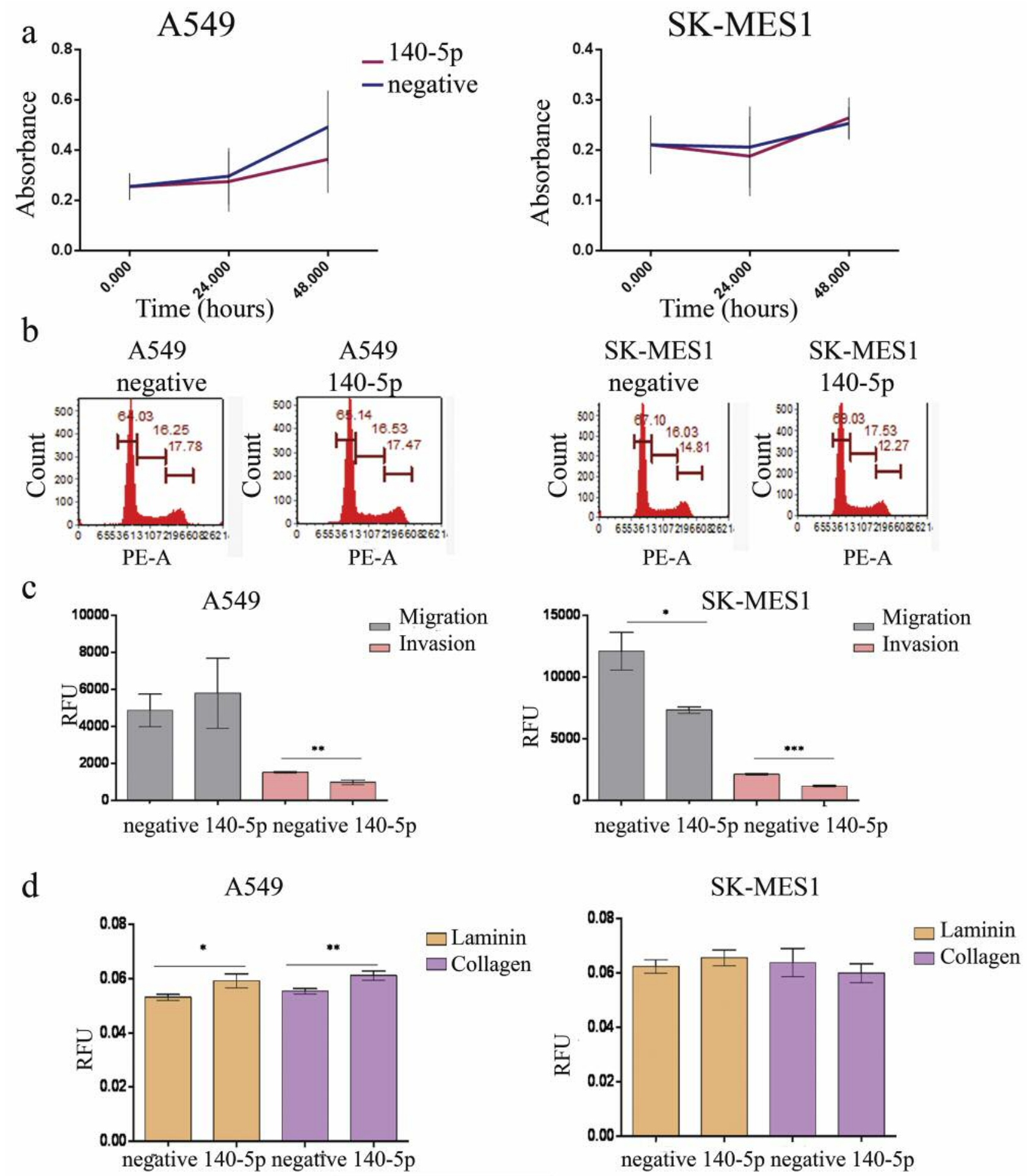

Figure 3. Effect of miR-140-5p replacement treatment on cancer cell behaviour. a) MiR-140-5p had no effect on the proliferation of A549 and SKMES1 cell lines. Data are presented as mean $\pm S D$ of three individual experiments undertaken in triplicate. $t$-Test was used to assess significance. $b$ ) The cell cycle of NSCLC cells was not influenced by the overexpression of miR-140-5p.c) MiR-140-5p reduced the migration and invasion ability of the SK-MES1 cells (on the right) and the motility of the A549 cell line (on the left). Data are presented as mean \pm SEM of three individual experiments undertaken in triplicate. $t$-Test was used to assess significance with $* p<0.05$, $* * p<0.01$ and $* * * p<0.001 . d)$ MiR-140-5p increased significantly the adhesion of the A549 cells to laminin and collagen, whereas no effect was observed in SK-MES-1. Data are presented as mean \pm SEM, three individual experiments undertaken in triplicate. $t$-Test was used to assess significance with $* p<0.05$ and $* * p<0.01$. 
a
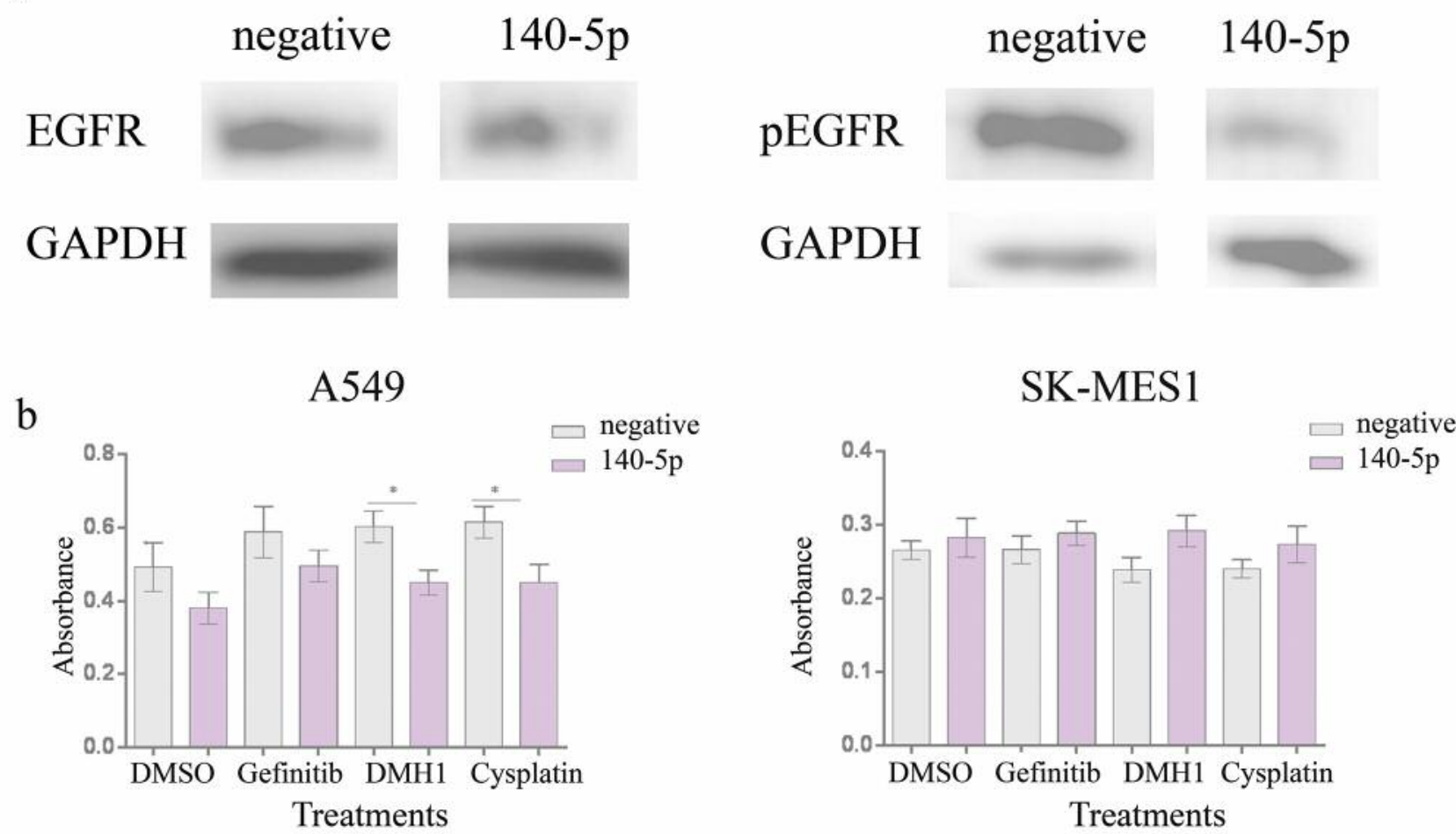

c

DMSO

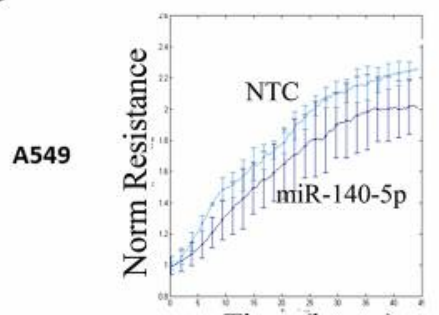

Time (hours)
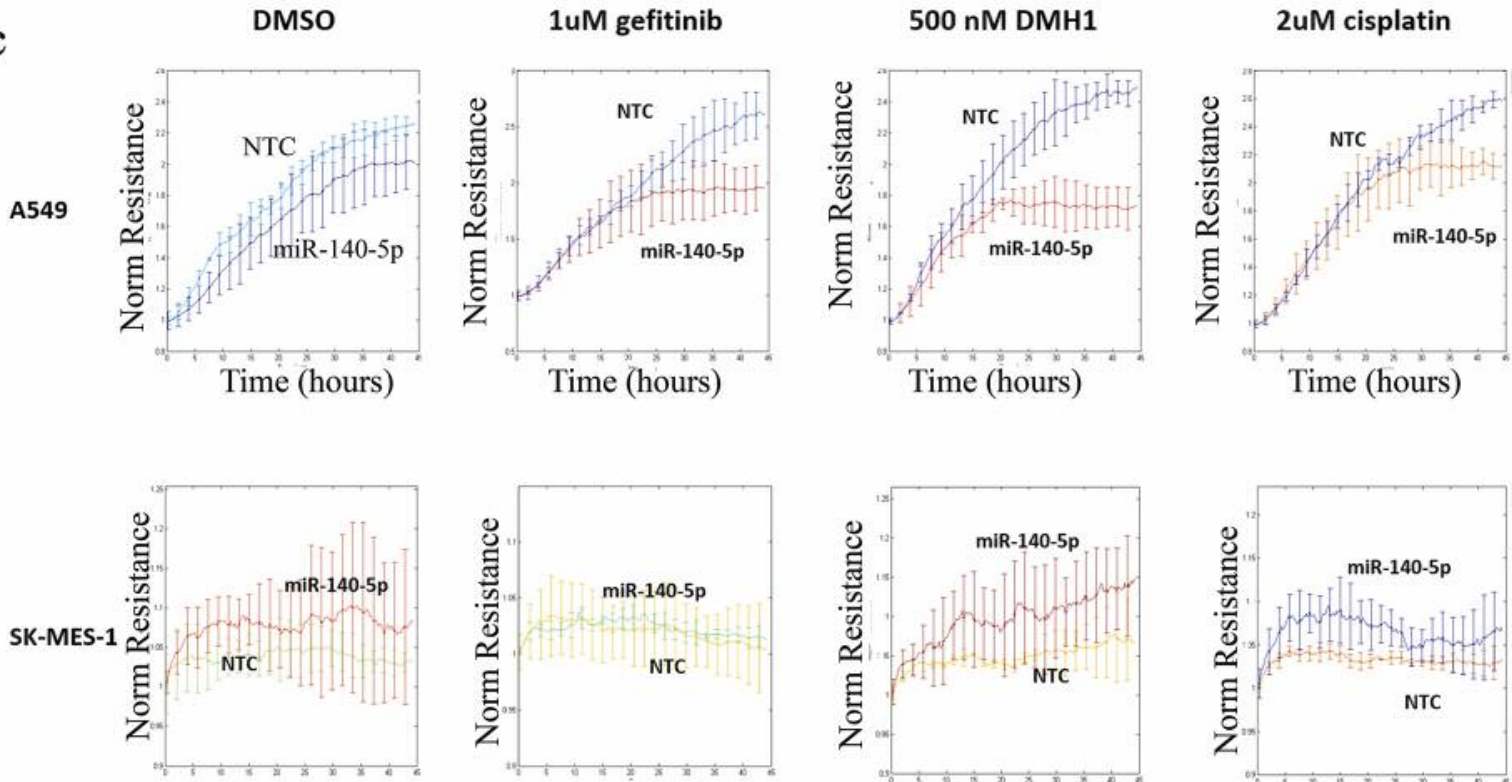

Time (hours)

Time (hours)
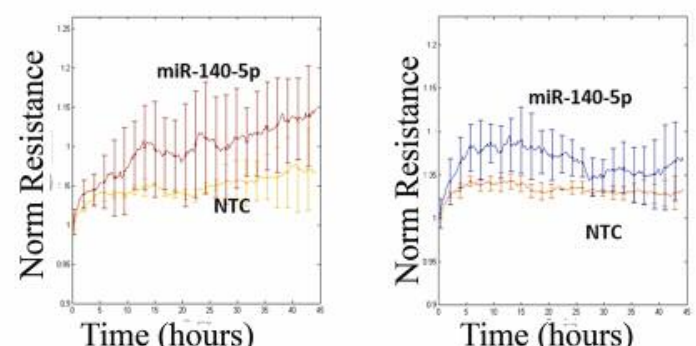

Figure 4. Effect of the combined treatment with miR-140-5p mimics and chemotherapeutics on NSCLC behaviour. a) MiR-140-5p decreased the protein level of EGFR and the active form pEGFR in the A549 cell line. b) The combined treatment with miR-140-5p mimics and some common chemotherapeutics did not have any effect on the SK-MES1 cell line, whereas miR-140-5p and DMH1 and cisplatin decreased the proliferation of the A549 cells. Data are presented as mean $\pm S E M$ of three individual experiments undertaken in triplicate. $t$-Test was used to assess significance with $\left.{ }^{*} p<0.05 . c\right)$ The migration ability of NSCL cells was investigated in real time by monitoring the resistance of the lung cancer cell monolayer over a range of frequencies $(1,000 \mathrm{~Hz}-64,000 \mathrm{~Hz})$. After $24 \mathrm{~h}$, the lung cancer monolayers were electrically wounded (2000 $\mathrm{mA}$ for $20 \mathrm{~s}$ each. A549 was inhibited by the simultaneous treatment with miR-140-5p mimics and, alternatively, DMH1 and cisplatin, whereas no effect was observed in SKMES1. Data are presented as mean \pm SEM of three individual experiments undertaken in triplicate. $t$-Test was used to assess significance with $* p<0.05$. 


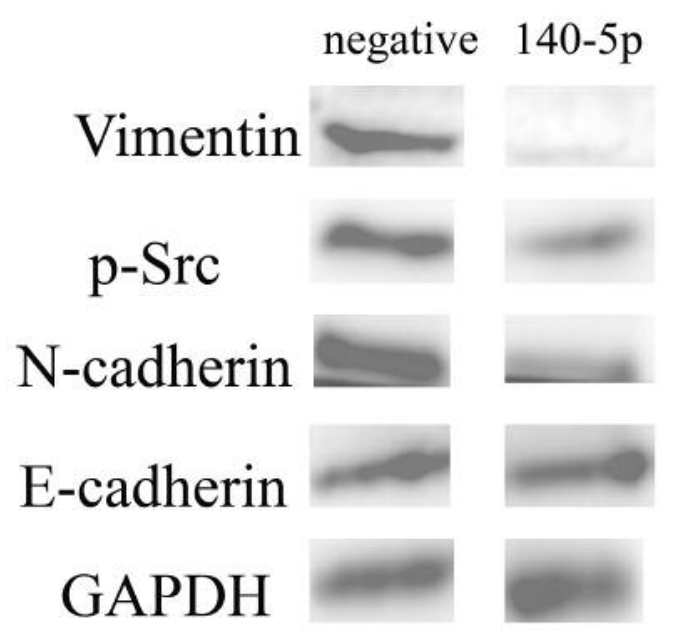

Figure 5. Western blot of $A 549$ cells $48 \mathrm{~h}$ after transfection with miR140-5p mimics. Vimentin, $p$-src and $N$-cadherin were normally upregulated in cancer and miR-140-5p was able to decrease their expression. No difference was shown for E-cadherin expression, usually down-regulated in tumours, in A549 cells transfected with miR-140-5p.

in lung cancer and mutations in this gene were recorded in some patients from our cohort. We found that, when transfected into A549 cells, miR-140-5p reduced the levels of EGFR and its active phosphorylated form pEGFR protein (Figure 4a). EGFR receptor tyrosine kinase inhibitor (TKI) Gefitinib is widely used in clinical practice as a treatment for NSCLC patients and our results showed that miR-140-5p combined with Gefinitib reduced the migration ability of the A549 cells $(p<0.05)$ (Figure $4 c) . A L K 2$ mutations are also associated with NSCLC progression and our data on the proliferation and cell migration of NSCLC cells treated with miR-140-5p mimics and DMH1 demonstrated that miR-140-5p boosts the inhibitory activity of this drug on both tumour cell proliferation $(p<0.05)$ and migration in A549 $(p<0.05)$ (Figures $4 b$ and $c$ ). Finally, miR-140-5p together with Cisplatin, reduced the cell proliferation of A549 (Figure $4 b)$. No significant effect with any treatment was observed in SK-MES1 (Figure 4b and c).

MiR-140-5p inhibits the Epithelial Mesenchymal Transition (EMT) in NSCLC through unknown pathways. The previous results suggested an involvement of miR-140-5p in the invasion of NSCLC. To test this hypothesis, we investigated the effect of miR-140-5p on some key factors involved in EMT, using western blot in A549 cells. The levels of the Vimentin, $\mathrm{p}$-Src and $\mathrm{N}$-cadherin decreased in cells transfected with miR-140-5p mimics $48 \mathrm{~h}$ after transfection, whereas no differences were seen in the levels of E-cadherin compared to the negative control (Figure 5).

\section{Discussion}

We confirmed that 140-5p is down-regulated in NSCLC, in both immortalised cell lines and tissue samples from NSCLC patients. MiRNA replacement using specific mimics reduced the migration and invasion of lung cancer cells and also improved the adhesive properties of the A549 tumour cells. However, there was no effect on proliferation and cell cycle. We also demonstrated that miR-140-5p enhanced the effect of some anti-cancer reagents in use for the treatment of the NSCLC. Finally, we verified that miR$140-5 p$ is involved in EMT, as some key molecules in this process were down-regulated by miR-140-5p, namely vimentin, $\mathrm{N}$-cadherin and $\mathrm{p}$-Src.

MiRNAs are small molecules that play an important regulatory role in both physiological and pathological processes. MiRNAs in tumours can act as onco-suppressors when their expression is lower than in normal conditions. Their low molecular weight and small size, together with their ability to target multiple molecules without toxicity, provides a new opportunity for cancer treatment compared to other methods such as gene therapy. MiRNA replacement therapy aims at substituting tumour suppressed miRNAs by using oligonucleotide mimics containing the same sequence as the mature endogenous miRNA. This could be achieved by using vectors (plasmids or lentiviruses) expressing miRNA precursors or by delivering artificial miRNA duplexes (mimics). So far, the use of miRNA mimics in therapeutics has been successful in both in vitro and in vivo models (14-16). MiR-140-5p downregulation in NSCLC, the most common form of lung cancer, contributes to the progression of the malignancy $(4,6)$.

In this study we restored the miR-140-5p in two NSCLC cell lines by delivering miRNA mimics with the use of a lipid-based transfection technology and studied the effects on cancer cell behaviour. Moreover, we evaluated whether miR-140-5p could act synergistically with some chemotherapeutics currently in use for the clinical treatment of NSCLC patients. We showed that miR-140-5p mimics are able to significantly increase the levels of miR-140-5p compared to the control. During transient transfection, the levels of the transfected miRNA were time-dependent. By monitoring the cells at two time points, we observed the highest miR-140-5p levels $24 \mathrm{~h}$ after transfection in A549 cells. Interestingly, in the SK-MES1 cells miR-140-5p expression increased after $24 \mathrm{~h}$ and further increased $48 \mathrm{~h}$ after transfection, though we did not continue to explore this trend beyond $48 \mathrm{~h}$. We also evaluated the efficacy of the miR-140-5p mimic on the NSCLC cell behaviour in vitro. The transfection of miR-140-5p into A549 cells reduced the invasive properties of the cancer cells as well as increasing their attachment to the main components of the ECM (laminin and collagen). In SK-MES1 cells, miR-140-5p 
decreased the invasive properties of the cells, though did not appear to alter their ECM adherence capabilities. The combined treatment of miRNA mimics with some commercially available chemotherapeutic agents for the treatment of NSCLC boosted their efficacy in preventing the progression of the malignancy. We focused on the effect of gefinitib, DMH1 and cisplatin. EGFR is overexpressed in many tumours, including NSCLC, where the gene is mutated in approximately $10-35 \%$ of all cases (13). Its constitutive activation leads to the progression of the malignancy and correlates with reduced survival, lymph node metastasis and poor chemo-sensitivity (17). As with the EGFR pathway the bone morphogenetic protein (BMP) signalling cascade is constitutively activated in NSCLC. Evidence has demonstrated that the BMP Type I Receptor antagonists prevent the disease progression $(18,19)$. The BMPs are growth factors that belong to the transforming growth factor beta (TGF- $\beta$ ) superfamily and bind their transmembrane serine/threonine kinases receptors on the cell surface (type I and type II receptors). The ALK2 receptor is a BMP type I receptor and although the mutations in the $A L K 2$ gene are less frequent than the ones in EGFR they account for approximately $3-7 \%$ of all lung cancer cases. The drugs included in this study were gefinitib, an EGFR inhibitor, DMH1, an ALK2 inhibitor and cisplatin. All of them have therefore been approved and currently in use for lung cancer patients (13). The inhibitory role of EGFR and ALK2 inhibitors in NSCLC invasion is well known, but here we tested whether their activity could be enhanced by the addition of miR-140-5p mimics. We found that miR-140-5p together with DMH1 or cisplatin reduced the proliferation of A549 cells, an effect that was not observed with miR-140$5 \mathrm{p}$ replacement standalone treatment. In A549 cells the combination of miR-140-5p and gefinitib or DMH1 also decreased the migratory ability of the cancer cells in vitro. In contrast the combined treatment with miR-140-5p mimics and chemotherapeutics did not have any effect on the SKMES1 cells.

As miR-140-5p reduced the invasive properties of the A549 cells, we tested its effect on the expression of some factors involved in EMT. We found that miR-140-5p reduced the expression of the active and inactive forms of EGFR, as well as vimentin, the phospho-proto-oncogene tyrosineprotein kinase (p-Src) and $\mathrm{N}$-cadherin, thus confirming its involvement in the progression of NSCLC.

Taking all together, our data demonstrate the role of miR$140-5 p$ as an onco-suppressor in NSCLC. Further studies are necessary to understand which pathways are influenced by the miR-140-5p replacement treatment and which mRNAs are specifically targeted. Additionally, nanoparticles able to deliver miR-140-5p in vivo will be required to better understand the potential role of this miRNA as therapeutic agent for the treatment of NSCLC.

\section{Conflicts of Interest}

The Authors declare no conflicts of interest.

\section{Acknowledgements}

The Authors would like to thank the support from Cardiff China Medical Research Collaborative (Cardiff, United Kingdom) and Cancer Research Wales.

\section{References}

1 Ferlay J, Shin HR, Bray F, Forman D, Mathers C and Parkin DM: Estimates of worldwide burden of cancer in 2008: GLOBOCAN 2008. International journal of cancer 127: 28932917, 2010.

2 Ferlay J, Soerjomataram I, Dikshit R, Eser S, Mathers C, Rebelo M, Parkin DM, Forman D and Bray F: Cancer incidence and mortality worldwide: sources, methods and major patterns in GLOBOCAN 2012. International journal of cancer 136: E359E386, 2015.

3 Zhang B, Pan X, Cobb GP and Anderson TA: microRNAs as oncogenes and tumor suppressors. Developmental biology 302: 1-12, 2007.

4 Yuan Y, Shen Y, Xue L and Fan H: miR-140 suppresses tumor growth and metastasis of non-small cell lung cancer by targeting insulin-like growth factor 1 receptor. PloS one 8: e73604, 2013.

5 Dong W, Yao C, Teng X, Chai J, Yang X and Li B: MiR-140-3p suppressed cell growth and invasion by downregulating the expression of ATP8A1 in non-small cell lung cancer. Tumour biology: the journal of the International Society for Oncodevelopmental Biology and Medicine 37: 2973-2985, 2016.

$6 \mathrm{Li} \mathrm{W}$ and He F: Monocyte to macrophage differentiationassociated (MMD) targeted by miR-140-5p regulates tumor growth in non-small cell lung cancer. Biochemical and biophysical research communications 450: 844-850, 2014.

7 Yang H, Fang F, Chang R and Yang L: MicroRNA-140-5p suppresses tumor growth and metastasis by targeting transforming growth factor $\beta$ receptor 1 and fibroblast growth factor 9 in hepatocellular carcinoma. Hepatology 58: 205-217, 2013.

8 Li W, Jiang G, Zhou J, Wang H, Gong Z, Zhang Z, Min K, Zhu $\mathrm{H}$ and Tan Y: Down-Regulation of miR-140 Induces EMT and Promotes Invasion by Targeting Slug in Esophageal Cancer. Cellular Physiology and Biochemistry 34: 1466-1476, 2014.

9 Wei Q, Liu J, Wang N, Zhang X, Jin J, Chin-Sang I, Zheng J and Jia Z: Structures of an Eph receptor tyrosine kinase and its potential activation mechanism. Acta Crystallogr D Biol Crystallogr 70: 3135-3143, 2014.

10 Kota J, Chivukula RR, O'Donnell KA, Wentzel EA, Montgomery CL, Hwang HW, Chang TC, Vivekanandan P, Torbenson M, Clark KR, Mendell JR and Mendell JT: Therapeutic microRNA delivery suppresses tumorigenesis in a murine liver cancer model. Cell 137: 1005-1017, 2009.

11 Xue W, Dahlman JE, Tammela T, Khan OF, Sood S, Dave A, Cai W, Chirino LM, Yang GR, Bronson R, Crowley DG, Sahay G, Schroeder A, Langer R, Anderson DG and Jacks T: Small RNA combination therapy for lung cancer. Proceedings of the National Academy of Sciences of the United States of America 111: E3553-3561, 2014. 
12 Li QJ, Chau J, Ebert PJ, Sylvester G, Min H, Liu G, Braich R, Manoharan M, Soutschek J, Skare P, Klein LO, Davis MM and Chen CZ: miR-181a is an intrinsic modulator of $\mathrm{T}$ cell sensitivity and selection. Cell 129: 147-161, 2007.

$13 \mathrm{https}: / / \mathrm{www}$.mycancergenome.org/content/disease/lung-cancer/.

14 Chen Y, Zhu X, Zhang X, Liu B and Huang L: Nanoparticles modified with tumor-targeting scFv deliver siRNA and miRNA for cancer therapy. Molecular therapy: the journal of the American Society of Gene Therapy 18: 1650-1656, 2010.

15 Choi KY, Silvestre OF, Huang X, Hida N, Liu G, Ho DN, Lee S, Lee SW, Hong JI and Chen X: A nanoparticle formula for delivering siRNA or miRNAs to tumor cells in cell culture and in vivo. Nature protocols 9: 1900-1915, 2014.

16 Hsu SH, Yu B, Wang X, Lu Y, Schmidt CR, Lee RJ, Lee LJ, Jacob ST and Ghoshal K: Cationic lipid nanoparticles for therapeutic delivery of siRNA and miRNA to murine liver tumor. Nanomedicine: nanotechnology, biology, and medicine 9 : 1169-1180, 2013.
17 Bethune G, Bethune D, Ridgway N and Xu Z: Epidermal growth factor receptor (EGFR) in lung cancer: an overview and update. Journal of thoracic disease 2: 48-51, 2011.

18 Langenfeld E, Hong CC, Lanke G and Langenfeld J: Bone morphogenetic protein type I receptor antagonists decrease growth and induce cell death of lung cancer cell lines. PLoS One 8: e61256, 2013.

19 Hao J, Lee R, Chang A, Fan J, Labib C, Parsa C, Orlando R, Andresen B and Huang Y: DMH1, a small molecule inhibitor of BMP type i receptors, suppresses growth and invasion of lung cancer. PLoS One 9: e90748, 2014.

Received May 2, 2017

Revised May 19, 2017

Accepted May 25, 2017 